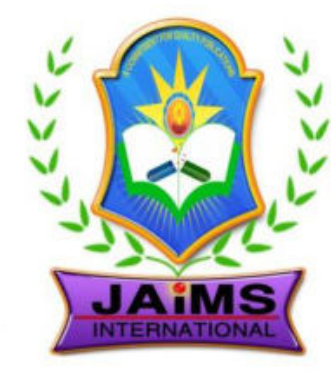

ISSN 2456-3110

Vol $5 \cdot$ Issue 1

Jan-Feb 2020

Journal of

Ayurveda and Integrated Medical Sciences

www.jaims.in

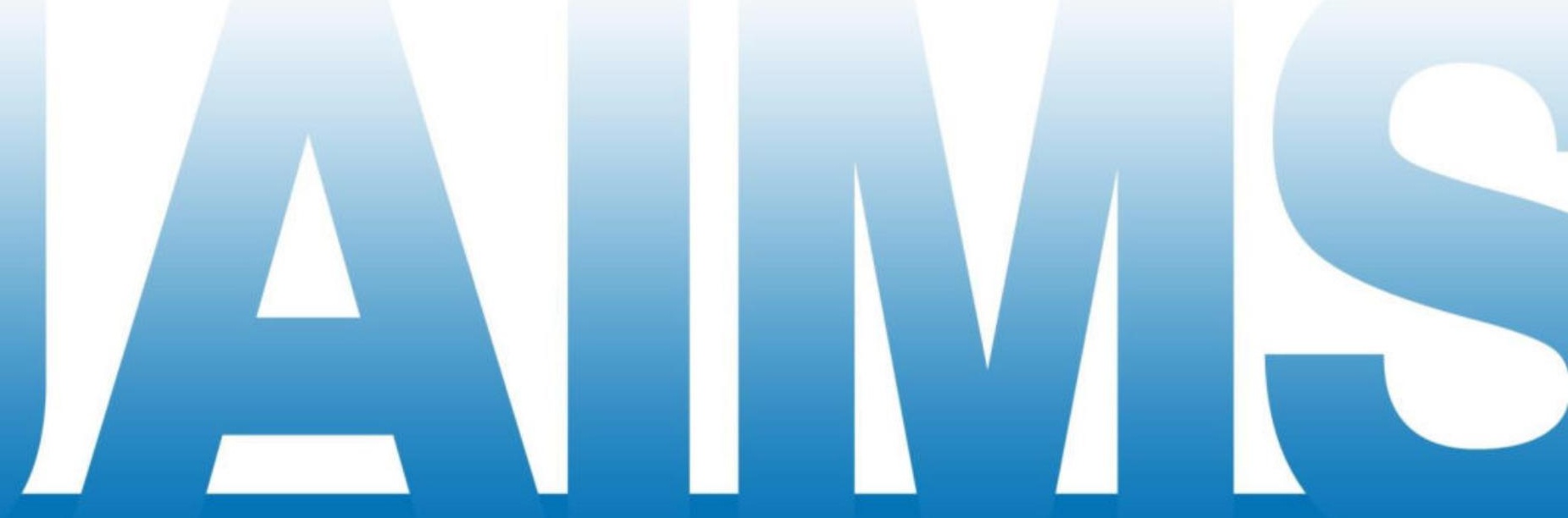

An International Journal for Researches in Ayurveda and Allied Sciences

\title{
Charaka
}




\title{
Ayurveda and Integrated Medical Sciences
}

\section{A comparative clinical study to evaluate the efficacy of Ashwagandha Ghrita on Growth and Development of Infants}

\author{
Dr. Koushik Baishya ${ }^{1}$, Prof. Rakesh Sharma², Dr. Vinod Kumar ${ }^{3}$ \\ ${ }^{1}$ Post Graduate Scholar, ${ }^{2}$ Professor \& HOD, ${ }^{3}$ Reader, P.G. Department of Kaumarbhritya, Rajiv Gandhi Govt. P.G. \\ Ayurvedic Medical College \& Hospital Paprola, Distt.Kangra, Himachal Pradesh, INDIA.
}

\section{A B S TR A C T}

\begin{abstract}
Introduction: Growth and development are unique characteristics of children. Growth is defined as an increase in the size of an individual due to increase in number and size of the cells, where development refers to qualitative and quantitative changes and acquisition of a variety of competencies for functioning optimally in a social milieu. The present study has been planned to evaluate the effect of Ashwagandhaghrita on physical growth and development of infants. Aims \& objectives: To study the efficacy of Ashwagandha Ghrita on Growth and development of infants, in terms of change occurred in anthropometry and milestones during the study period. Materials \& methods: The study was conducted on 40 healthy infants for a period of 12 weeks and a case performa was filled with the data obtained by interrogation, physical examination and collection of details of each child. Results: Ashwagandha Ghrita showed better effect on physical growth in terms of increment in weight, head circumference, chest circumference, mid arm circumference, crown to heel length as well as early significant achievement of developmental milestones compared to control group. Conclusion: Ashwagandha Ghrita has proved to be beneficial on Growth and Development of infants.
\end{abstract}

Key words: Growth, Development, Ashwagandha Ghrita, Infants.

\section{INTRODUCTION}

Growth and development are the normal biological phenomenon of all living beings. They are unique characteristics of children and any obstacle in this process at any stage can possibly result in aberration. Growth is an essential feature of life of a child that

Address for correspondence:
Dr. Koushik Baishya
Post Graduate Scholar, P.G. Department of Kaumarbhritya, Rajiv
Gandhi Govt. P.G. Ayurvedic Medical College \& Hospital Paprola,
Distt.Kangra, Himachal Pradesh, INDIA.
E-mail: kb564127@gmail.com
Submission Date: 22/12/2019 Accepted Date: 28/01/2020
Quick Response Code

distinguishes him or her from an adult. The process starts from the time of conception and continues until maturity. It is defined as an increase in the size of an individual due to increase in number and size of the cells, resulting in an overall increase. Development refers to qualitative and quantitative changes and acquisition of a variety of competencies for functioning optimally in a social milieu. It depends on maturation and myelination of brain; unless that has occurred, no amount of practice can make the child learn that skill. It may be stressed that, besides $10 \%$ prevalence of developmental delay, the early identification of such problems remains difficult. Although severe disorders can be recognized in infancy, it is unusual to diagnose speech impairment, hyperactivity or emotional disorders before the age of 3 or 4 years; learning disabilities are rarely recognized before children start their schooling. If one can diagnose developmental delay in early stages of growth, the intervention can reduce long term sequel. 
The child acquires different skills and talent at different There are different factors explained in Ayurvedic classics which are going for the maintenance and promotion of growth and development in children. These factors, influencing growth and development can be classified as Garbhapoorvaavastha (prior to conception), Garbhakaleenaavastha (during pregnancy) and Prasavaottaraavastha (after birth). In our classical texts Lehan karma is advised to enhance energy, intellect and to protect the child from infections.For lehan karma many compounds have been prescribed in Ayurvedic texts. Ashwagandha ghrita is one of them. Therefore, this study is planned to evaluate the effect of Ashwagandha ghrita on physical growth and development of infants, in terms of change occur in anthropometry and milestones during the study period.

\section{AIMS AND OBJECTIVES}

1. To study the efficacy of Ashwagandha Ghrita on Growth and development of infants, in terms of change occurred in anthropometry and milestones during the study period.

2. To evaluate the safety of the trial drug in infants.

\section{Materials AND Methods}

For the present study, infants were selected from IPD/OPD of Department of Kaumarabhritya, R. G. G. P. G. Ayu. College \& Hospital, Paprola-Baijnath, Distt.Kangra, Himachal Pradesh. Total 40 healthy infants were registered and divided into two groups. Out of them, 6 were dropped out from the study because they never turn up on subsequent follow ups. A case proforma was filled with the data obtained by interrogation, physical examination and collection of details of each child.

\begin{tabular}{|l|l|}
\hline Inclusion criteria & Exclusion criteria \\
\hline $\begin{array}{l}\text { 1. Age eligibility - upto 1 } \\
\text { year }\end{array}$ & $\begin{array}{l}\text { 1. } \\
\text { Infant suffering from any } \\
\text { congenital, hereditary or } \\
\text { acute systemic illness }\end{array}$ \\
\hline 2. Gender eligibility - Both & 2. Infants not fulfilling the \\
\hline
\end{tabular}

\begin{tabular}{|c|c|c|c|}
\hline & male and female & & criteria of inclusion \\
\hline \multirow[t]{2}{*}{3.} & \multirow[t]{2}{*}{$\begin{array}{l}\text { Infants whose parents will } \\
\text { give their written } \\
\text { informed consent }\end{array}$} & 3. & $\begin{array}{l}\text { Infant who has been } \\
\text { dropped on subsequent } \\
\text { follow up }\end{array}$ \\
\hline & & & $\begin{array}{l}\text { Whose parents have } \\
\text { refused to participate in } \\
\text { the study }\end{array}$ \\
\hline
\end{tabular}

\section{Grouping of patients}

Study was conducted on 40 selected infants in two groups:

- Group A - In this group 20 infants were given the trial drug Ashwagandha Ghrita. 18 infants completed the trial and 2 were dropped out.

- Group B - No medication were given to the infants of this group. Out of 20 infants 16 infants completed the trial and 4 were dropped out.

Treatment schedule

\begin{tabular}{|l|l|l|}
\hline Particulars & Group A & Group B \\
\hline Drug & Ashwagandha Ghrita & No medication \\
\hline Dosage & $0.5 \mathrm{ml} / \mathrm{kg} / \mathrm{day}$ & - \\
\hline Anupana & Milk & - \\
\hline Duration & 4 weeks & 4 weeks \\
\hline
\end{tabular}

*Follow ups: 4 follow ups at 1, 4, 8 \& 12 weeks

\section{RESULTS}

\section{Assessment of drug response}

In each group, growth and development were assessed at four weeks i.e. 4, 8 \& 12 weeks interval by anthropometrical evaluation and changes occurred in achievement of age specific developmental milestones. Scoring system was adopted for assessment of different developmental milestones at different ages with grades from zero to two, according to the appearance of different developmental milestones at different ages. ('0'= early; ' 1 '= timely; '2'= late) 
(First week follow up is to see any adverse effect of the trial drugs in infants.)

\section{Statistical analysis}

The obtained data was analyzed statistically and expressed in terms of mean, standard deviation (SD), $t$ value and $p$ values in paired \& unpaired ' $t$ ' test.

Assessment of various Anthropometrical Parameters at subsequent follow ups

Table 1: Comparison between groups R-FU2

\begin{tabular}{|l|l|l|l|l|l|}
\hline \multirow{2}{*}{$\begin{array}{l}\text { Anthropometric } \\
\text { parameters }\end{array}$} & \multicolumn{2}{|l|}{$\begin{array}{l}\text { Mean increment } \\
\text { (R-FU2) }\end{array}$} & $\begin{array}{l}\text { 't' } \\
\text { value }\end{array}$ & $\begin{array}{l}\text { P } \\
\text { value }\end{array}$ & Result \\
\cline { 2 - 5 } Gr. A & Gr. B & & \\
\hline Weight (Kg) & $\begin{array}{l}0.861 \\
( \pm 0.252)\end{array}$ & $\begin{array}{l}0.670 \\
( \pm 0.185)\end{array}$ & 2.492 & 0.018 & $\mathrm{~S}$ \\
\hline $\begin{array}{l}\text { Crown to heel } \\
\text { length (cm) }\end{array}$ & $\begin{array}{l}3.250 \\
( \pm 0.773)\end{array}$ & $\begin{array}{l}2.688 \\
( \pm 0.797)\end{array}$ & 2.087 & 0.045 & $\mathrm{~S}$ \\
\hline $\begin{array}{l}\text { Head } \\
\text { Circumference } \\
\text { (cm) }\end{array}$ & $\begin{array}{l}1.667 \\
( \pm 0.536)\end{array}$ & $\begin{array}{l}1.456 \\
( \pm 0.560)\end{array}$ & 1.119 & 0.271 & IS \\
\hline $\begin{array}{l}\text { Chest } \\
\text { Circumference } \\
\text { (cm) }\end{array}$ & $\begin{array}{l}2.008 \\
( \pm 0.652)\end{array}$ & $\begin{array}{l}1.700 \\
( \pm 0.610)\end{array}$ & 1.418 & 0.166 & IS \\
\hline $\begin{array}{l}\text { Mid Upper Arm } \\
\text { Circumference } \\
\text { (cm) }\end{array}$ & $\begin{array}{l}0.736 \\
( \pm 0.217)\end{array}$ & $\begin{array}{l}0.634 \\
( \pm 0.196)\end{array}$ & 1.427 & 0.163 & IS \\
\hline
\end{tabular}

$[\mathrm{R}=$ At the time of registration; FU2= Follow up 2; $\mathrm{S}=$ Significant; HS= Highly Significant; IS= Insignificant]

Table 2: Comparison between groups R-FU3

\begin{tabular}{|l|l|l|l|l|l|}
\hline \multirow{2}{*}{$\begin{array}{l}\text { Anthropometric } \\
\text { parameters }\end{array}$} & \multicolumn{2}{|l|}{$\begin{array}{l}\text { Mean increment } \\
\text { (R-FU3) }\end{array}$} & $\begin{array}{l}\text { 't' } \\
\text { value }\end{array}$ & $\begin{array}{l}\text { P } \\
\text { value }\end{array}$ & Result \\
\cline { 2 - 6 } & Gr. A & Gr. B & & \\
\hline Weight (Kg) & $\begin{array}{l}1.622 \\
( \pm 0.462)\end{array}$ & $\begin{array}{l}1.247 \\
( \pm 0.389)\end{array}$ & 2.544 & 0.016 & $\mathrm{~S}$ \\
\hline $\begin{array}{l}\text { Crown to heel } \\
\text { length(cm) }\end{array}$ & $\begin{array}{l}6.461 \\
( \pm 1.622)\end{array}$ & $\begin{array}{l}5.094 \\
( \pm 1.573)\end{array}$ & 2.488 & 0.018 & $\mathrm{~S}$ \\
\hline
\end{tabular}

\begin{tabular}{|l|l|l|l|l|l|}
\hline $\begin{array}{l}\text { Head } \\
\text { Circumference } \\
(\mathrm{cm})\end{array}$ & $\begin{array}{l}2.844 \\
( \pm 0.953)\end{array}$ & $\begin{array}{l}2.506 \\
( \pm 1.011)\end{array}$ & 1.004 & 0.323 & IS \\
\hline $\begin{array}{l}\text { Chest } \\
\text { Circumference } \\
(\mathrm{cm})\end{array}$ & $\begin{array}{l}3.806 \\
( \pm 1.110)\end{array}$ & $\begin{array}{l}3.206 \\
( \pm 1.213)\end{array}$ & 1.504 & 0.142 & IS \\
\hline $\begin{array}{l}\text { Mid Upper Arm } \\
\text { Circumference } \\
(\mathrm{cm})\end{array}$ & $\begin{array}{l}1.444 \\
( \pm 0.433)\end{array}$ & $\begin{array}{l}1.222 \\
( \pm 0.429)\end{array}$ & 1.502 & 0.143 & IS \\
\hline
\end{tabular}

$[\mathrm{R}=$ At the time of registration; FU3= Follow up 3; $\mathrm{S}=$ Significant; HS= Highly Significant; IS= Insignificant]

Table 3: Comparison between groups R-FU4

\begin{tabular}{|l|l|l|l|l|l|}
\hline \multirow{2}{*}{$\begin{array}{l}\text { Anthropometric } \\
\text { parameters }\end{array}$} & \multicolumn{2}{|l|}{$\begin{array}{l}\text { Mean increment } \\
\text { (R-FU4) }\end{array}$} & $\begin{array}{l}\text { 't' } \\
\text { value }\end{array}$ & $\begin{array}{l}\text { P } \\
\text { value }\end{array}$ & Result \\
\cline { 2 - 5 } & Gr. A & Gr. B & & \\
\hline Weight (Kg) & $\begin{array}{l}2.272 \\
( \pm 0.672)\end{array}$ & $\begin{array}{l}1.753 \\
( \pm 0.548)\end{array}$ & 2.449 & 0.020 & $\mathrm{~S}$ \\
\hline $\begin{array}{l}\text { Crown to heel } \\
\text { length (cm) }\end{array}$ & $\begin{array}{l}9.194 \\
( \pm 2.291)\end{array}$ & $\begin{array}{l}7.156 \\
( \pm 2.174)\end{array}$ & 2.652 & 0.012 & $\mathrm{~S}$ \\
\hline $\begin{array}{l}\text { Head } \\
\text { Circumference } \\
\text { (cm) }\end{array}$ & $\begin{array}{l}4.256 \\
( \pm 1.474)\end{array}$ & $\begin{array}{l}3.744 \\
( \pm 1.528)\end{array}$ & 0.993 & 0.328 & IS \\
\hline $\begin{array}{l}\text { Chest } \\
\text { Circumference } \\
\text { (cm) }\end{array}$ & $\begin{array}{l}5.483 \\
( \pm 1.542)\end{array}$ & $\begin{array}{l}4.550 \\
( \pm 1.756)\end{array}$ & 1.651 & 0.109 & IS \\
\hline $\begin{array}{l}\text { Mid Upper Arm } \\
\text { Circumference } \\
\text { (cm) }\end{array}$ & $\begin{array}{l}2.056 \\
( \pm 0.637)\end{array}$ & $\begin{array}{l}1.756 \\
( \pm 0.641)\end{array}$ & 1.364 & 0.182 & IS \\
\hline
\end{tabular}

$[R=$ At the time of registration; FU4= Follow up 4; $S=$ Significant; HS= Highly Significant; IS= Insignificant]

Assessment of various Developmental Domains after each subsequent follow ups

Scoring system was adopted for assessment of different developmental milestones at different ages with grades from zero to two, according to the appearance of different developmental milestones at different ages. ('0'= early; ' 1 '= timely; ' 2 '= late) 
Table 4: Assessment of development after $2^{\text {nd }}$ follow up

\begin{tabular}{|c|c|c|c|c|c|c|c|c|}
\hline \multirow{2}{*}{$\begin{array}{l}\text { Gro } \\
\text { ups }\end{array}$} & \multicolumn{2}{|c|}{ Mean } & \multirow{2}{*}{$\begin{array}{l}\% \\
\text { Improve } \\
\text { ment }\end{array}$} & \multirow{2}{*}{$\begin{array}{l}\text { S.D } \\
\pm\end{array}$} & \multirow{2}{*}{$\begin{array}{l}\text { S.E } \\
\pm\end{array}$} & \multirow{2}{*}{$\begin{array}{l}\text { 't' } \\
\text { val } \\
\text { ue }\end{array}$} & \multirow{2}{*}{$\begin{array}{l}P \\
\text { valu } \\
\text { e }\end{array}$} & \multirow{2}{*}{$\begin{array}{l}\text { Res } \\
\text { ult }\end{array}$} \\
\hline & $\mathbf{R}$ & $\begin{array}{l}\text { FU } \\
2\end{array}$ & & & & & & \\
\hline \multicolumn{9}{|c|}{ Gross motor development } \\
\hline \multirow[t]{2}{*}{ Gr A } & 1.7 & 1.0 & 41.9 & 0.4 & 0.1 & 6.6 & $<0.0$ & HS \\
\hline & 22 & 00 & & 61 & 09 & 48 & 01 & \\
\hline \multirow[t]{2}{*}{ Gr B } & 1.4 & 1.3 & 4.3 & 0.7 & 0.1 & 0.3 & 0.75 & IS \\
\hline & 38 & 75 & & 72 & 93 & 24 & 1 & \\
\hline \multicolumn{9}{|c|}{ Fine motor development } \\
\hline \multirow[t]{2}{*}{ Gr A } & 1.6 & 0.9 & 43.31 & 0.5 & 0.1 & 5.3 & $<0.0$ & HS \\
\hline & 67 & 44 & & 75 & 35 & 33 & 01 & \\
\hline \multirow[t]{2}{*}{ Gr B } & 1.3 & 1.4 & -4.5 & 0.6 & 0.1 & - & 0.71 & IS \\
\hline & 75 & 38 & & 80 & 70 & 0.3 & 8 & \\
\hline \multicolumn{9}{|c|}{ Language development } \\
\hline \multirow[t]{2}{*}{ Gr A } & 1.6 & 1.0 & 37.93 & 0.5 & 0.1 & 5.1 & $<0.0$ & HS \\
\hline & 11 & 00 & & 02 & 18 & 69 & 01 & \\
\hline \multirow[t]{2}{*}{ Gr B } & 1.5 & 1.4 & 4.13 & 0.6 & 0.1 & 0.3 & 0.71 & IS \\
\hline & 00 & 38 & & 80 & 70 & 68 & 8 & \\
\hline \multicolumn{9}{|c|}{ Social \& Adaptive development } \\
\hline \multirow[t]{2}{*}{ Gr A } & 1.6 & 1.0 & 36.65 & 0.5 & 0.1 & 5.1 & $<0.0$ & HS \\
\hline & 67 & 56 & & 02 & 18 & 69 & 01 & \\
\hline \multirow[t]{2}{*}{ Gr B } & 1.5 & 1.5 & 0.00 & 0.6 & 0.1 & 0.0 & 1.00 & IS \\
\hline & 00 & 00 & & 32 & 58 & 00 & 0 & \\
\hline
\end{tabular}

$[R=$ At the time of registration; FU2= Follow up 2; $S=$ Significant; HS= Highly Significant; IS= Insignificant]

Table 5: Intergroup comparison after $2^{\text {nd }}$ follow up

\begin{tabular}{|l|l|l|l|l|}
\hline \multicolumn{2}{|l|}{ Mean difference (R-FU2) } & \multirow{2}{*}{ t value } & P value & Result \\
\cline { 1 - 5 } Group A & Group B & & & \\
\hline \multicolumn{2}{|l|}{ Gross motor development } & & & \\
\hline 0.722 & 0.063 & 3.066 & 0.004 & $\mathrm{~S}$ \\
\hline
\end{tabular}

\begin{tabular}{|l|l|l|l|l|}
\hline \multicolumn{1}{|l|}{ Fine motor development } & 0.063 & 3.647 & $<0.001$ & HS \\
\hline 0.722 & 0.063 & 2.697 & 0.011 & HS \\
\hline Language development & \multicolumn{5}{|l|}{} \\
\hline 0.611 & 0.000 & 3.138 & 0.004 & $\mathrm{~S}$ \\
\hline Social \& adaptive development & & & \\
\hline 0.611 & &
\end{tabular}

[R= At the time of registration; FU2= Follow up 2; $S=$ Significant; HS= Highly Significant; IS= Insignificant]

Table 6: Assessment of development after $3^{\text {rd }}$ follow up

\begin{tabular}{|c|c|c|c|c|c|c|c|c|}
\hline \multirow{2}{*}{$\begin{array}{l}\text { Gro } \\
\text { ups }\end{array}$} & \multicolumn{2}{|c|}{ Mean } & \multirow{2}{*}{$\begin{array}{l}\% \\
\text { Improve } \\
\text { ment }\end{array}$} & \multirow{2}{*}{$\begin{array}{l}\text { S.D } \\
\pm\end{array}$} & \multirow{2}{*}{$\begin{array}{l}\text { S.E } \\
\pm\end{array}$} & \multirow{2}{*}{$\begin{array}{l}\text { 't' } \\
\text { val } \\
\text { ue }\end{array}$} & \multirow{2}{*}{$\begin{array}{l}P \\
\text { valu } \\
\text { e }\end{array}$} & \multirow{2}{*}{$\begin{array}{l}\text { Res } \\
\text { ult }\end{array}$} \\
\hline & $\mathbf{R}$ & $\begin{array}{l}\text { FU } \\
3\end{array}$ & & & & & & \\
\hline \multicolumn{9}{|c|}{ Gross motor development } \\
\hline \multirow[t]{2}{*}{ Gr A } & 1.7 & 0.6 & 61.26 & 0.5 & 0.1 & 8.3 & $<0.0$ & HS \\
\hline & 22 & 67 & & 39 & 27 & 04 & 01 & \\
\hline \multirow[t]{2}{*}{ Gr B } & 1.4 & 1.2 & 13.07 & 0.6 & 0.1 & 1.1 & 0.27 & IS \\
\hline & 38 & 50 & & 55 & 64 & 45 & 0 & \\
\hline \multicolumn{9}{|c|}{ Fine motor development } \\
\hline \multirow[t]{2}{*}{ Gr A } & 1.6 & 0.7 & 56.69 & 0.5 & 0.1 & 7.4 & $<0.0$ & HS \\
\hline & 67 & 22 & & 39 & 27 & 30 & 01 & \\
\hline \multirow[t]{2}{*}{ Gr B } & 1.3 & 1.1 & 13.6 & 0.5 & 0.1 & 1.3 & 0.18 & IS \\
\hline & 75 & 88 & & 44 & 36 & 79 & 8 & \\
\hline \multicolumn{9}{|c|}{ Language development } \\
\hline \multirow[t]{2}{*}{ Gr A } & 1.6 & 0.5 & 65.5 & 0.5 & 0.1 & 8.3 & $<0.0$ & HS \\
\hline & 11 & 56 & & 39 & 27 & 04 & 01 & \\
\hline \multirow[t]{2}{*}{ Gr B } & 1.5 & 1.3 & 12.5 & 0.7 & 0.1 & 1.0 & 0.33 & IS \\
\hline & 00 & 13 & & 50 & 88 & 00 & 3 & \\
\hline \multicolumn{9}{|c|}{ Social \& Adaptive development } \\
\hline \multirow[t]{2}{*}{ Gr A } & 1.6 & 0.5 & 66.65 & 0.5 & 0.1 & 8.0 & $<0.0$ & HS \\
\hline & 67 & 56 & & 83 & 37 & 86 & 01 & \\
\hline Gr B & 1.5 & 1.3 & 12.5 & 0.7 & 0.1 & 1.0 & 0.33 & IS \\
\hline
\end{tabular}


\begin{tabular}{l|l}
00 & 13
\end{tabular}

\begin{tabular}{l|l|l|l}
\hline 50 & 88 & 00 & 3
\end{tabular}

$[R=$ At the time of registration; FU3= Follow up 3; $S=$ Significant; HS= Highly Significant; IS= Insignificant]

Table 7: Intergroup comparison after $3^{\text {rd }}$ follow up

\begin{tabular}{|l|l|l|l|l|}
\hline \multicolumn{2}{|l|}{ Mean difference (R-FU3) } & t value & P value & Result \\
\hline Group A & Group B & & \\
\hline Gross motor development & 0.188 & 4.236 & $<0.001$ & HS \\
\hline 1.056 & 0.188 & 4.069 & $<0.001$ & HS \\
\hline Fine motor development & 0.188 & 3.907 & $<0.001$ & HS \\
\hline 0.944 & 0.188 & 4.033 & $<0.001$ & HS \\
\hline Language development & \multicolumn{5}{|l}{} \\
\hline 1.056 & Social \& adaptive development \\
\hline 1.111 &
\end{tabular}

$[R=$ At the time of registration; FU3= Follow up 3; $S=$ Significant; HS= Highly Significant; IS= Insignificant]

Table 8: Assessment of development after $4^{\text {th }}$ follow up

\begin{tabular}{|l|l|l|l|l|l|l|l|l|}
\hline \multirow{2}{*}{$\begin{array}{l}\text { Gro } \\
\text { ups }\end{array}$} & \multicolumn{2}{|l|}{ Mean } & $\%$ & S.D & S.E & $\begin{array}{l}\text { 't' } \\
\text { yal }\end{array}$ & $\begin{array}{l}\text { P } \\
\text { valu }\end{array}$ & $\begin{array}{l}\text { Res } \\
\text { ult }\end{array}$ \\
\cline { 2 - 6 } & R & FU & $\begin{array}{l}\text { Improve } \\
\text { ment }\end{array}$ & \pm & \pm & $\begin{array}{l}\text { val } \\
\text { ue }\end{array}$ & \\
\hline
\end{tabular}

Gross motor development

\begin{tabular}{|l|l|l|l|l|l|l|l|l|}
\hline Gr A & $\begin{array}{l}1.7 \\
22\end{array}$ & $\begin{array}{l}0.2 \\
78\end{array}$ & 83.8 & $\begin{array}{l}0.5 \\
11\end{array}$ & $\begin{array}{l}0.1 \\
21\end{array}$ & $\begin{array}{l}11 . \\
99\end{array}$ & $\begin{array}{l}<0.0 \\
01\end{array}$ & HS \\
\hline Gr B & $\begin{array}{l}1.4 \\
38\end{array}$ & $\begin{array}{l}1.2 \\
50\end{array}$ & 13.07 & $\begin{array}{l}0.7 \\
50\end{array}$ & $\begin{array}{l}0.1 \\
88\end{array}$ & $\begin{array}{l}1.0 \\
00\end{array}$ & $\begin{array}{l}0.33 \\
3\end{array}$ & IS \\
\hline
\end{tabular}

Fine motor development

\begin{tabular}{|l|l|l|l|l|l|l|l|l|}
\hline Gr A & $\begin{array}{l}1.6 \\
67\end{array}$ & $\begin{array}{l}0.3 \\
33\end{array}$ & 80.0 & $\begin{array}{l}0.5 \\
94\end{array}$ & $\begin{array}{l}0.1 \\
40\end{array}$ & $\begin{array}{l}9.5 \\
22\end{array}$ & $\begin{array}{l}<0.0 \\
01\end{array}$ & HS \\
\hline Gr B & $\begin{array}{l}1.3 \\
75\end{array}$ & $\begin{array}{l}1.1 \\
88\end{array}$ & 13.6 & $\begin{array}{l}0.7 \\
50\end{array}$ & $\begin{array}{l}0.1 \\
88\end{array}$ & $\begin{array}{l}1.0 \\
00\end{array}$ & $\begin{array}{l}0.33 \\
3\end{array}$ & IS \\
\hline
\end{tabular}

Language development

\begin{tabular}{|l|l|l|l|l|l|l|l|l|}
\hline Gr A & $\begin{array}{l}1.6 \\
11\end{array}$ & $\begin{array}{l}0.3 \\
89\end{array}$ & 75.8 & $\begin{array}{l}0.5 \\
48\end{array}$ & $\begin{array}{l}0.1 \\
29\end{array}$ & $\begin{array}{l}9.4 \\
57\end{array}$ & $\begin{array}{l}<0.0 \\
01\end{array}$ & HS \\
\hline Gr B & $\begin{array}{l}1.5 \\
00\end{array}$ & $\begin{array}{l}1.2 \\
50\end{array}$ & 16.7 & $\begin{array}{l}0.8 \\
56\end{array}$ & $\begin{array}{l}0.2 \\
14\end{array}$ & $\begin{array}{l}1.1 \\
68\end{array}$ & $\begin{array}{l}0.26 \\
1\end{array}$ & IS \\
\hline
\end{tabular}

Social \& Adaptive development

\begin{tabular}{|l|l|l|l|l|l|l|l|l|}
\hline Gr A & $\begin{array}{l}1.6 \\
67\end{array}$ & $\begin{array}{l}0.3 \\
33\end{array}$ & 80.0 & $\begin{array}{l}0.5 \\
94\end{array}$ & $\begin{array}{l}0.1 \\
40\end{array}$ & $\begin{array}{l}9.5 \\
22\end{array}$ & $\begin{array}{l}<0.0 \\
01\end{array}$ & HS \\
\hline Gr B & $\begin{array}{l}1.5 \\
00\end{array}$ & $\begin{array}{l}1.3 \\
13\end{array}$ & 12.5 & $\begin{array}{l}0.8 \\
34\end{array}$ & $\begin{array}{l}0.2 \\
09\end{array}$ & $\begin{array}{l}0.8 \\
99\end{array}$ & $\begin{array}{l}0.38 \\
3\end{array}$ & IS \\
\hline
\end{tabular}

$[R=$ At the time of registration; FU4= Follow up 4; $S=$ Significant; HS= Highly Significant; IS= Insignificant]

Table 9: Intergroup comparison after $4^{\text {th }}$ follow up

\begin{tabular}{|c|c|c|c|c|}
\hline \multicolumn{2}{|c|}{ Mean difference (R-FU4) } & \multirow[t]{2}{*}{$t$ value } & \multirow[t]{2}{*}{$P$ value } & \multirow[t]{2}{*}{ Result } \\
\hline Group A & Group B & & & \\
\hline \multicolumn{5}{|c|}{ Gross motor development } \\
\hline 1.444 & 0.301 & 5.766 & $<0.001$ & HS \\
\hline \multicolumn{5}{|c|}{ Fine motor development } \\
\hline 1.333 & 0.188 & 4.965 & $<0.001$ & HS \\
\hline \multicolumn{5}{|c|}{ Language development } \\
\hline 1.222 & 0.250 & 3.988 & $<0.001$ & HS \\
\hline \multicolumn{5}{|c|}{ Social \& adaptive development } \\
\hline 1.333 & 0.188 & 4.653 & $<0.001$ & HS \\
\hline
\end{tabular}

$[R=$ At the time of registration; FU4= Follow up 4; $S=$ Significant; HS= Highly Significant; IS= Insignificant]

\section{DISCUSSION}

After the completion of trial, Ashwagandha Ghrita is found to be beneficial in growth parameters like weight \& crown heel length and also shows significant results on Development of infants compared to control group.

Ashwagandha Ghrita possesses Pushtikrit and Balavardhan properties. The table below shows the contents of Ashwagandhaghrita along with their Karma and pharmacological actions:

\begin{tabular}{|l|l|l|}
\hline Ingredients & Karma & $\begin{array}{l}\text { Action based on } \\
\text { pharmacological studies }\end{array}$ \\
\hline Ashwagandha & Balya, Rasayan & $\begin{array}{l}\text { Antibiotic activity } \\
\text { (Withaferin A), } \\
\text { immunomodulator, } \\
\text { cognition enhancing \& } \\
\text { memory improving effect, } \\
\text { anti-oxidant (sitoindosides }\end{array}$ \\
\hline
\end{tabular}




\begin{tabular}{|l|l|l|}
\hline \multirow{5}{*}{ Go-dugdha } & $\begin{array}{l}\text { VII-X, Withaferin A, } \\
\text { glycowithanolides), } \\
\text { Rasayana, } \\
\text { Budhhipravodhaka }\end{array}$ & $\begin{array}{l}\text { Energy, micronutrients } \\
\text { (calcium, phosphorus, } \\
\text { iron), vitamins (Vit. A, } \\
\text { Thiamine, Riboflavin, } \\
\text { Niacin, Vit. C) }\end{array}$ \\
\hline Go-ghrita & $\begin{array}{l}\text { Deepana, } \\
\text { Rochaka, } \\
\text { Rasayana, Balya, } \\
\text { Brimhana, } \\
\text { Yogavahi, } \\
\text { Medhya, } \\
\text { Vayasthapaka, } \\
\text { Rasa-Shukra- } \\
\text { Ojovardhaka }\end{array}$ & $\begin{array}{l}\text { Anti-oxidant (Vit. E, beta- } \\
\text { carotene), 8\% lower } \\
\text { saturated fatty acid which } \\
\text { makes it easily digestible }\end{array}$ \\
\hline
\end{tabular}

\section{CONCLUSION}

The growth rate of an infant is perhaps a better indicator of general health and nutritional status. From the above study we can conclude that Ashwagandha Ghrita enhances growth and development in the infant. Though, breast milk is best for optimal growth and development during infancy, this formulation may be used as an adjuvant to get timely development of milestones with better growth. No adverse effect of the trial drug was observed during the study.

\section{REFERENCES}

1. Kasyapa Samhita with Vidyotini Hindi Commentary by Sri Satyapal Bhisagacharya, Chaukhamba Sanskrit Sansthan, Varanasi, 2015

2. Yogratnakar with Vidyotni commentary, Chaukambha Sanskrit sansthan by Bhisagratna Brahmashankar Shastri, Balrog Chikitsa.

3. Pt. Kashinath Shastri and Dr. Gorakhanath Chaturvedi, Charaka Samhita of Charaka with Vidyotini Hindi Commentary, Sutrasthana, Reprint edition; 2013 Chapter 13. Verses 14.
4. Sushruta Samhita by Kaviraj Ambikadutta Shastri, Chaukhamba Sanskrit Sansthan, Varanasi, 2012, Sutrasthan, Chapter 45/96.

5. Kaiyadeva Nighantu, edited and translated by Prof. Priyavrata Sharma \&Dr. Guru Prasada Sharma, Chaukhamba Orientalia, Varanasi, 2009, page 193

6. Rajnighantu, Chaukambha Prakashan by InderDev Tripathi 3rd Edition, Kshiradi varga,verse no. 10; 2003.

7. IAP Text Book of Pediatrics, Parthasarathy A editor, Jaypee Brothers Medical Publishers (P) Ltd, $6^{\text {th }}$ edition, 2016

8. Ghai Essential Pediatrics, editors: Vinod K Paul, Arvind Bagga, CBS Publishers \& Distributors Pvt Ltd, $8^{\text {th }}$ edition, reprint: 2017

9. MR Khan \& M Ekhlasur Rahman, Essence of Pediatrics, 4th Edition, Elsevier Publication, 2011

10. Nelson Textbook of Pediatrics, Robert M. Kliegman, Bonita F Stanton, Joseph W St Geme III, Nina F Schor. 1st South Asia Edition, Vol-1, RELX India Private Limited, New Delhi, 2016

11. Chris Plauche Johnson, Peter A. Blasco; Infant Growth and Development. Pediatrics in Review, Vol. 18, No. 7, July 1997

12. https://en.m.wikipedia.org/wiki/infant

13. A Review on Pharmacological Profile of Withania somnifera (Ashwagandha); Research and Reviews: Journal of Botanical Sciences; Volume 2, Issue 4, October-December, 2013

14. Shardul Chavan: Various Aspects of Ghrita with Special Reference to Cow Ghee; ayurpub 2017;II(1):342-346

15. Chawre Sushil Vilas, Shinde Vandana Sitaram, Kabra P. R., Raut Deokumar, Bhivgade H. S. Therapeutic and nutritional significance of Dugdha (Milk) with reference to cow's milk. Int. Res. J. Pharm. 2014; 5(6):449-453

How to cite this article: Dr. Koushik Baishya, Prof. Rakesh Sharma, Dr. Vinod Kumar. A comparative clinical study to evaluate the efficacy of Ashwagandha Ghrita on Growth and Development of Infants. J Ayurveda Integr Med Sci 2020;1:31-36. http://dx.doi.org/10.21760/jaims.5.1.7

Source of Support: Nil, Conflict of Interest: None declared. 